Supplement of Biogeosciences Discuss., 12, 4245-4272, 2015

http://www.biogeosciences-discuss.net/12/4245/2015/

doi:10.5194/bgd-12-4245-2015-supplement

(C) Author(s) 2015. CC Attribution 3.0 License.

(c) (1)

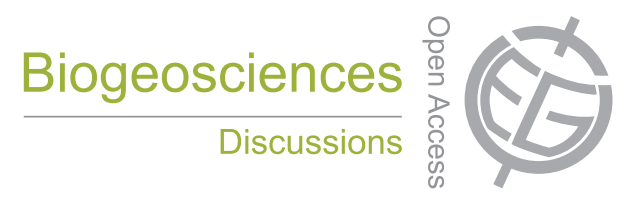

Supplement of

\title{
Convergent modeling of past soil organic carbon stocks but divergent projections
}

\section{Z. Luo et al.}

Correspondence to: Z. Luo (zhongkui.luo@csiro.au) and E. Wang (enli.wang@ csiro.au) 


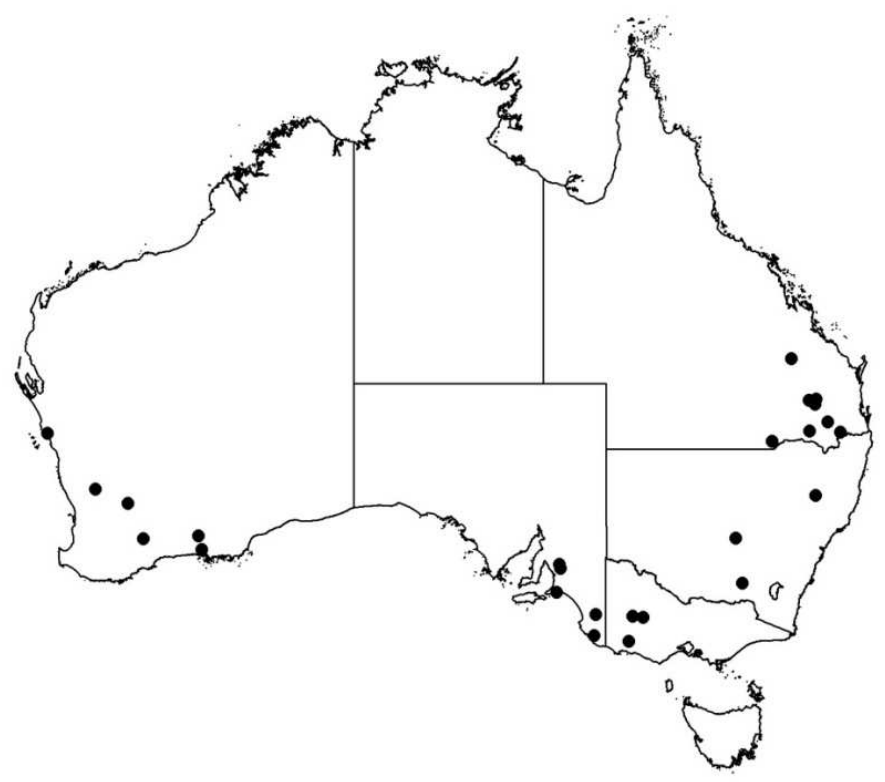

Supplement Figure 1. The geographical distribution of the 28 experimental sites where data are used in this study. See Table 1 in the supplement for details of the management and climate conditions at these sites. 


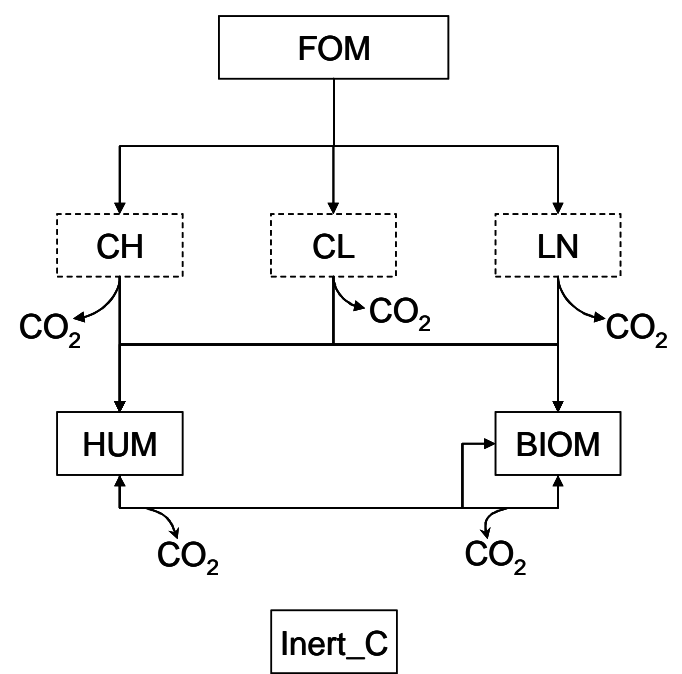

Supplement Figure 2. The transformation of soil organic carbon (SOC) pools in APSIM. FOM - fresh organic matter, $\mathrm{CH}$ - carbohydrate-like FOM, CL - cellulose-like FOM, LNlignin-like FOM, HUM - humus, BIOM - microbial biomass and products, Inert_C - inert SOC. 


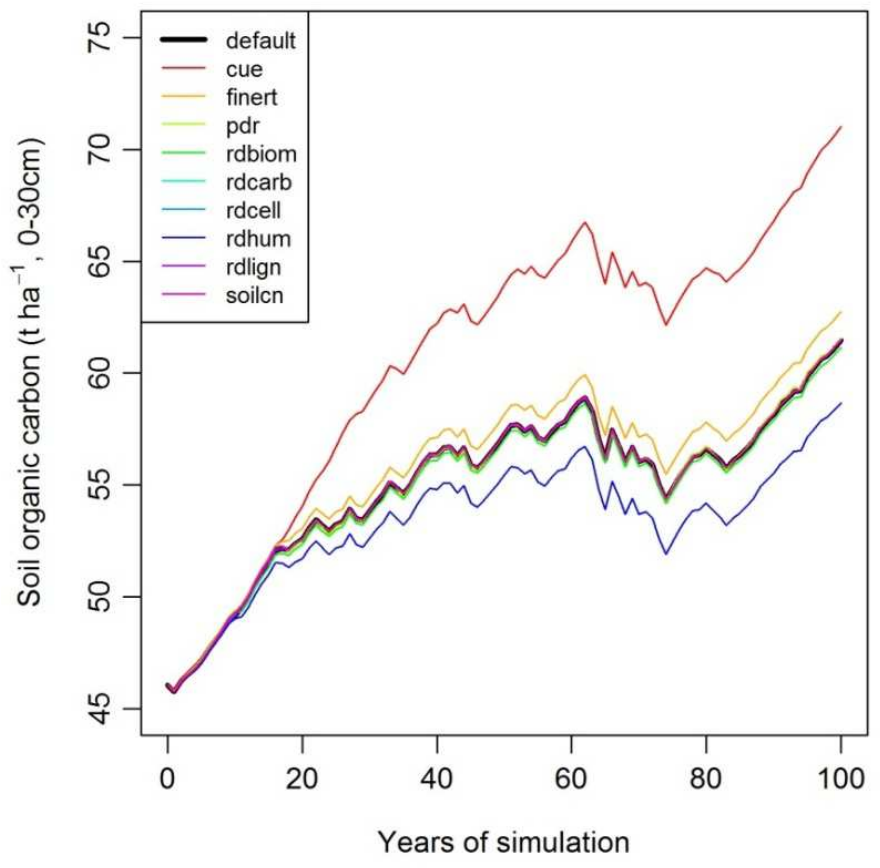

Supplement Figure 3. Sensitivity of modeled soil organic carbon to different model parameters. Simulated soil organic carbon dynamics under a $10 \%$ increase of a parameter value (color lines) is compared with that using default value (black bold line) under a continuous wheat system. See Table 2 in the supplement for the meaning of the legends. 

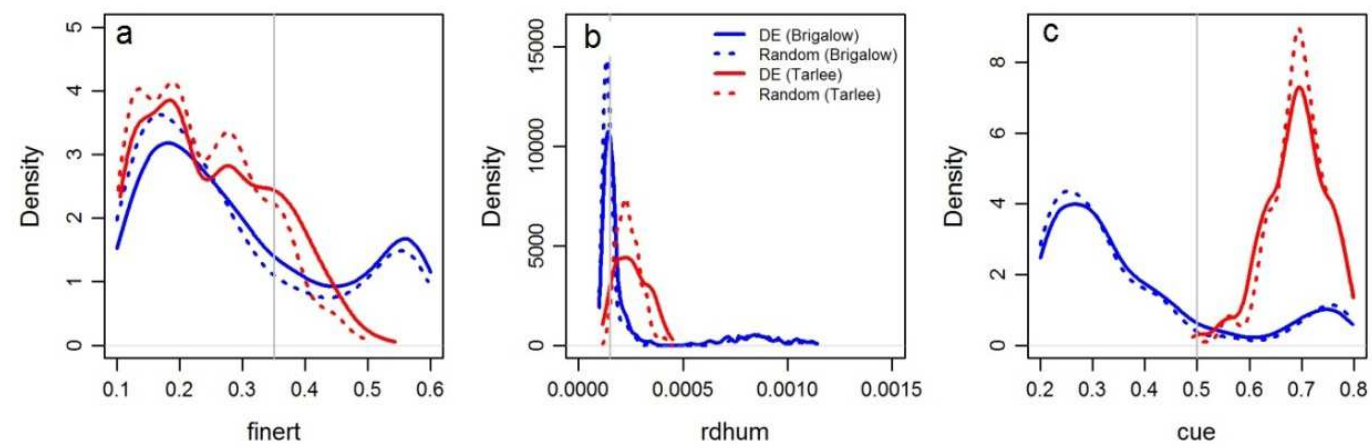

Supplement Figure 4. Posterior distributions of the three most important parameters at Brigalow and Tarlee of Australia. a, finert - fraction of inert organic carbon in total soil organic carbon. b, rdhum - potential decomposition rate of humic organic carbon. c, cue - microbial carbon use efficiency. The distribution derived using the differential evolution optimization (DE) are compared with that derived using the random sampling (Random) approach. Grey vertical lines show the default values of the three parameters in the model. See more details in the Methods section for the two optimization approaches. See Fig. $2 c$ and $d$ for the relationship of the three parameters at Tarlee and Brigalow respectively. 

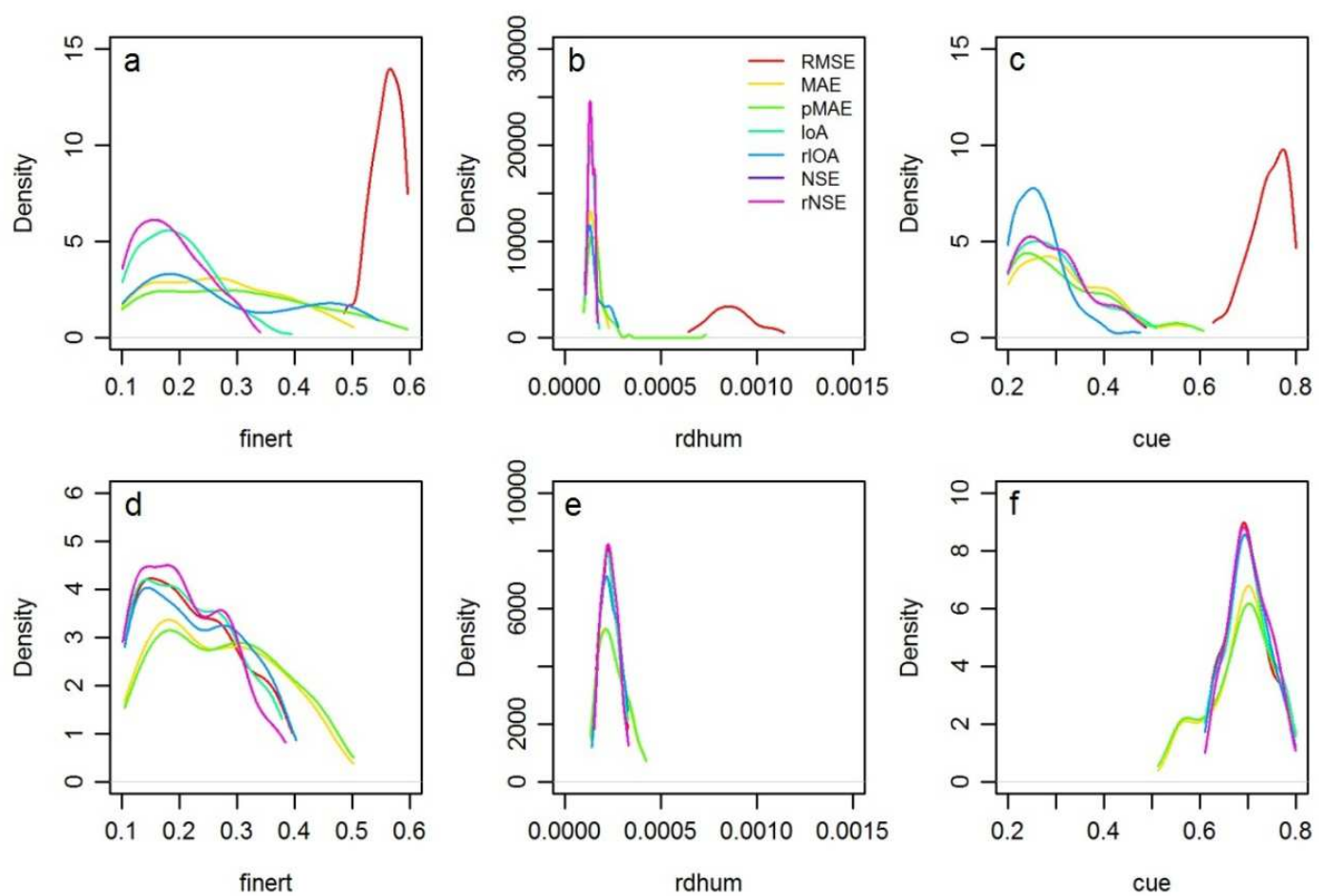

Supplement Figure 5. The effect of selection criterions on the optimized posterior distributions of model parameters (finert, rdhum and cue) at Brigalow ( $a, b$ and $c$ ) and Tarlee (d, e and f) of Australia. See Table 3 in the supplement for the seven criteria showed in the legend. 
Supplement Table 1. Climate conditions and management of the studied experiments in Australia. Data adopted from Skjemstad and Spouncer (2003).

\begin{tabular}{|c|c|c|c|}
\hline Location & $\begin{array}{l}\text { Rainfall } \\
(\mathrm{mm})\end{array}$ & $\begin{array}{l}\text { Temperature } \\
\left({ }^{\circ} \mathrm{C}\right)\end{array}$ & Management $^{*}$ \\
\hline Brigalow, Qld & 686 & 21.1 & W/S-R-Z-0-3-18 \\
\hline \multirow[t]{3}{*}{ Warra Field Trial, Qld } & 655 & 19.2 & $\mathrm{~W} / \mathrm{F}-\mathrm{R}-\mathrm{C}-0-1-13$ \\
\hline & & & $\mathrm{W} / \mathrm{P} / \mathrm{F}-\mathrm{R}-\mathrm{C}-75-1-13$ \\
\hline & & & $\mathrm{W} / \mathrm{F}-\mathrm{R}-\mathrm{C}-75-1-13$ \\
\hline \multirow[t]{3}{*}{ Hermitage, Qld } & 649 & 16.4 & $\mathrm{~W}-\mathrm{B}-\mathrm{C}-50-1-19$ \\
\hline & & & W-B-Z-50-1-19 \\
\hline & & & $\mathrm{W}-\mathrm{R}-\mathrm{C}-50-1-19$ \\
\hline \multirow[t]{2}{*}{ Langlands-Logie, Qld } & 655 & 19.7 & $\mathrm{~W}-\mathrm{R}-\mathrm{Z}-0-1-46$ \\
\hline & & & $\mathrm{W} / \mathrm{S}-\mathrm{R}-\mathrm{Z}-0-1-21$ \\
\hline \multirow[t]{2}{*}{ Warra, Qld } & 660 & 19.3 & W/S-R-0-1-66 \\
\hline & & & $\mathrm{W} / \mathrm{P}-\mathrm{R}-0-1-66$ \\
\hline \multirow[t]{3}{*}{ Cecilvale, Qld } & 655 & 19.6 & $\mathrm{~W} / \mathrm{F}-\mathrm{R}-0-1-36$ \\
\hline & & & $\mathrm{W}-\mathrm{R}-0-1-6$ \\
\hline & & & $\mathrm{W} / \mathrm{S}-\mathrm{R}-0-1-9$ \\
\hline \multirow[t]{3}{*}{ Waco, Qld } & 655 & 19.6 & $\mathrm{~W} / \mathrm{S} / \mathrm{F}-\mathrm{R}-0-1-70$ \\
\hline & & & $\mathrm{W}-\mathrm{R}-0-2-2$ \\
\hline & & & $\mathrm{W} / \mathrm{S}-\mathrm{R}-0-1-19$ \\
\hline \multirow[t]{2}{*}{ Thallon, Qld } & 499 & 20.4 & $\mathrm{~W}-\mathrm{R}-0-1-4$ \\
\hline & & & $\mathrm{W} / \mathrm{S}-\mathrm{R}-0-1-20$ \\
\hline \multirow[t]{2}{*}{ Billa Billa, Qld } & 585 & 19.3 & W/S-R-0-2-(25, 20) \\
\hline & & & $\mathrm{W} / \mathrm{P}-\mathrm{R}-0-1-20$ \\
\hline Condobolin, NSW & 427 & 17.6 & W-B-Z-0-1-15 \\
\hline \multirow[t]{2}{*}{ Tamworth, NSW } & 665 & 17.2 & $\mathrm{~W} / \mathrm{S} / \mathrm{F}-\mathrm{R}-\mathrm{Z}-0-2-30$ \\
\hline & & & $\mathrm{W} / \mathrm{S} / \mathrm{P}-\mathrm{R}-\mathrm{Z}-0-2-30$ \\
\hline
\end{tabular}




\begin{tabular}{|c|c|c|c|}
\hline Wagga Wagga, NSW & 522 & 16.0 & $\mathrm{~W}-\mathrm{R}-\mathrm{Z}-0-1-12$ \\
\hline Hamilton, Vic & 692 & 13.0 & $\mathrm{P}-\mathrm{R}-\mathrm{Z}-0-3-17$ \\
\hline \multirow[t]{2}{*}{ Horsham, Vic } & 439 & 14.8 & $\mathrm{~W} / \mathrm{F}-\mathrm{R}-\mathrm{Z}-0-2-83$ \\
\hline & & & $\mathrm{W} / \mathrm{F} / \mathrm{P}-\mathrm{R}-\mathrm{Z}-0-2-83$ \\
\hline Glenorchy, Vic & 426 & 14.6 & $\mathrm{~W} / \mathrm{O} / \mathrm{P} / \mathrm{F}-\mathrm{R}-\mathrm{Z}-0-1-23$ \\
\hline \multirow[t]{4}{*}{ Tarlee, SA } & 462 & 16.9 & $\mathrm{~W}-\mathrm{R}-\mathrm{Z}-0-1-18$ \\
\hline & & & W/S-R-Z-0-1-18 \\
\hline & & & W/F-R-Z-0-1-18 \\
\hline & & & W/P-R-Z-0-1-18 \\
\hline Freeling, SA & 463 & 15.9 & B/P-R-Z-0-1-27 \\
\hline Padthaway, SA & 517 & 14.8 & W/P-R-Z-0-1-24 \\
\hline Glencoe, SA & 770 & 13.8 & $\mathrm{P}-\mathrm{R}-\mathrm{Z}-0-1-24$ \\
\hline \multirow[t]{5}{*}{ Chapman, WA } & 457 & 19.6 & $\mathrm{~W}-\mathrm{R}-\mathrm{Z}-0-1-23$ \\
\hline & & & $\mathrm{P}-\mathrm{R}-\mathrm{Z}-0-1-23$ \\
\hline & & & $\mathrm{W} / \mathrm{P} 1-\mathrm{R}-\mathrm{Z}-0-1-23$ \\
\hline & & & $\mathrm{W} / \mathrm{P} 2-\mathrm{R}-\mathrm{Z}-0-1-23$ \\
\hline & & & W/P3-R-Z-0-1-23 \\
\hline \multirow[t]{5}{*}{ East Beverley, WA } & 369 & 18.2 & $\mathrm{~W}-\mathrm{R}-\mathrm{Z}-0-1-7$ \\
\hline & & & $\mathrm{P}-\mathrm{R}-\mathrm{Z}-0-1-7$ \\
\hline & & & W/P1-R-Z-0-1-7 \\
\hline & & & W/P2-R-Z-0-7 \\
\hline & & & W/P3-R-Z-0-1-7 \\
\hline \multirow[t]{4}{*}{ Gibson, WA } & 517 & 16.0 & W-R-Z-0-1-18 \\
\hline & & & $\mathrm{P}-\mathrm{R}-\mathrm{Z}-0-1-18$ \\
\hline & & & P/W/L1-R-Z-0-1-18 \\
\hline & & & P/W/L2-R-Z-0-1-18 \\
\hline
\end{tabular}


Merredin (heavy), WA 333

Merredin (light), WA

333

Newdegate, WA

Salmon Gums, WA

352

370

356

Wongan Hills, WA
17.8

P-R-Z-0-1-18

W/P1-R-Z-0-1-18

W/P2-R-Z-0-1-18

W/P3-R-Z-0-1-18

W/P4-R-Z-0-1-18

17.8

W-R-Z-0-1-11

W/P1-R-Z-0-1-11

W/P2-R-Z-0-1-11

W/P3-R-Z-0-1-11

W-R-C-0-1-11

W/P1-R-C-0-1-11

W/P2-R-C-0-1-11

W/P3-R-C-0-1-11

16.2

W-R-Z-0-1-21

P-R-Z-0-1-21

W/P1-R-Z-0-1-21

W/P2-R-Z-0-1-21

W/P3-R-Z-0-1-21

16.0

$\mathrm{W}-\mathrm{R}-\mathrm{C}-0-1-14$

$\mathrm{W} / \mathrm{P} 1-\mathrm{R}-\mathrm{C}-0-1-14$

W/P2-R-C-0-1-14

18.2

$\mathrm{W} / \mathrm{P} 2-\mathrm{R}-\mathrm{C}-0-2-6$

* Rotation (i.e., W/S)-Residue (B for burned or removed, $\mathrm{R}$ for retained)-Tillage (C for conventional tillage, $\mathrm{Z}$ for no-till)-Fertilizer (e.g., 50 for $50 \mathrm{~kg} \mathrm{~N} \mathrm{ha}^{-1} \mathrm{yr}^{-1}$ )-Soil types (e.g., 2 for the experiment was conducted under two soil types)-Experimental duration (e.g., 10 for 10 years). B, barely; W, wheat; O, oats; S, sorghum; P, pasture; F, fallow; L, lupin. 
Supplement Table 2. Model parameters analyzed for the sensitivity analysis in the study.

\begin{tabular}{llll}
\hline Parameter (unit) & $\begin{array}{l}\text { Default value used for } \\
\text { sensitivity analysis }\end{array}$ & $\begin{array}{l}\text { Importance } \\
\text { index }\end{array}$ & $\begin{array}{l}\text { Prior distribution } \\
\text { [min, max] }\end{array}$ \\
\hline cue (unitless) & 0.5 & 64.43 & {$[0.2,0.8]$} \\
finert (unitless) & 0.35 & 8.93 & {$[0.1,0.6]$} \\
$\operatorname{pdr}\left(\right.$ day $\left.^{-1}\right)$ & 0.1 & 0.96 & - \\
rdbiom $\left(\right.$ day $\left.^{-1}\right)$ & 0.0081 & 2.23 & - \\
rdcarb $\left(\right.$ day $\left.^{-1}\right)$ & 0.2 & 0.012 & - \\
rdcell $\left(\right.$ day $\left.^{-1}\right)$ & 0.05 & 0.088 & - \\
rdlign $\left(\right.$ day $\left.^{-1}\right)$ & 0.0095 & 0.0097 & - \\
rdhum (day & -1 \\
soilcn $\left(\right.$ unitless $\left.^{-1}\right)$ & 0.00015 & 18.79 & {$[0.000015,0.0015]$} \\
\hline
\end{tabular}

"cue: microbial carbon use efficiency (unitless); finert: initial fraction of inert organic matter in total SOC (unitless); pdr: potential decomposition rate for aboveground FOM $\left(\right.$ day $\left.^{-1}\right)$; rdbiom: potential decomposition rate for microbial biomass; rdcarb: potential decomposition rate for carbohydrate-like $\mathrm{C}$ in belowground FOM; rdcell: potential decomposition rate for cellulose-like $\mathrm{C}$ in belowground FOM; rdlign: potential decomposition rate for lignin-like $\mathrm{C}$ in belowground FOM; rdhum: potential decomposition rate for humic organic matter; soilcn: carbon to nitrogen ratio of soil. 
Supplement Table 3. Performance measures for comparing model predictions and observations.

\begin{tabular}{lcc}
\hline Criterion & Formulation $^{*}$ & Range $^{\dagger}$ \\
\hline Root mean square error (RMSE) & $\sqrt{\frac{\sum_{i=1}^{N}\left(P_{i}-O_{i}\right)^{2}}{N}}$ & {$[\mathbf{0},+\infty]$} \\
Mean absolute error (MAE) & $\frac{\sum_{i=1}^{N}\left|P_{i}-O_{i}\right|}{N}$ & {$[\mathbf{0},+\infty]$} \\
Percentage MAE (pMAE) & $\frac{\sum_{i=1}^{N} \mid \frac{P_{i}-O_{i} \mid}{O_{i} \mid}}{N}$ & {$[\mathbf{0},+\infty]$} \\
Index of agreement (IoA) & $\frac{\sum_{i=1}^{N}\left(P_{i}-O_{i}\right)^{2}}{\sum_{i=1}^{N}\left(\frac{\left.\left|P_{i}-\bar{P}\right|+\left|O_{i}-\bar{O}\right|\right)^{2}}{O_{i}}\right)^{2}}$ & {$[\mathbf{1},+\infty]$} \\
Relative IoA (rIoA) & {$[\mathbf{1},+\infty]$} \\
\cline { 2 - 3 } & $\frac{\sum_{i=1}^{N}\left(\frac{P_{i}-O_{i}}{O_{i}}\right.}{\sum_{i=1}^{N}\left(\frac{\left|P_{i}-\bar{P}\right|+\left|O_{i}-\bar{O}\right|}{\bar{O}}\right)^{2}}$ & \\
Nash-Sutcliffe Efficiency (NSE) & $\frac{\sum_{i=1}^{N}\left(P_{i}-O_{i}\right)^{2}}{\sum_{i=1}^{N}\left(P_{i}-\bar{O}\right)^{2}}$ & {$[\mathbf{1},+\infty]$} \\
Relative NSE (rNSE) & $\frac{\sum_{i=1}^{N}\left(\frac{P_{i}-O_{i}}{O_{i}}\right)^{2}}{\sum_{i=1}^{N}\left(\frac{P_{i}-\bar{O}}{\bar{O}}\right)^{2}}$ & {$[\mathbf{1},+\infty]$} \\
\hline
\end{tabular}

${ }^{*} P_{i}$ and $O_{i}$ denote the predicted and observed value $i ; \bar{O}$ is the mean of observed values. ${ }^{\dagger}$ The bold number shows the target value when reach perfect match, i.e., no difference between predicted and observed values. 